\title{
A facial EMG data analysis for emotion classification based on spectral kurtogram and CNN
}

\section{Vikram Kehri* and R.N. Awale}

\author{
Electrical Engineering Department, \\ VJTI, \\ H R Mahajani Marg, Matunga East, Mumbai, 400019, India \\ Email: vakehri@vjti.org.in \\ Email: rnawale@el.vjti.ac.in \\ *Corresponding author
}

\begin{abstract}
The current digital signal processing techniques are not completely effective because of the presence of different noises, added during the acquisition of facial electromyogram (FEMG) signals, and therefore, FEMG data analysis needs strong artificial intelligent techniques along with the advanced signal processing tools. A novel approach for the emotion classification from FEMG signals is proposed in this paper which is based on the kurtogram analysis and convolutional neural network $(\mathrm{CNN})$. Kurtogram is time-frequency energy density pattern gives additional information regarding frequency contents. In this work, the kurtogram considered as an input vector introduced to CNN for emotion classification. The different levels of kurtogram are considered to find the best feature set for an efficient result. Facial EMGs for five different facial emotions were recorded using two sensor wireless data acquisition device. 93\% classification accuracy was achieved using our proposed method. The classification results show that the proposed method effectively differentiates the emotion class compares to other methods (SVM, ANN, and $\mathrm{KNN}$ ).
\end{abstract}

Keywords: facial electromyogram; FEMG; human machine interface; HMI; convolution neural network; CNN; support vector machine; SVM; artificial neural network; ANN; K-nearest neighbours; KNN.

Reference to this paper should be made as follows: Kehri, V. and Awale, R.N. (2020) 'A facial EMG data analysis for emotion classification based on spectral kurtogram and CNN', Int. J. Digital Signals and Smart Systems, Vol. 4, Nos. 1/2/3, pp.50-63.

Biographical notes: Vikram Kehri received his BE in Electronics and Telecommunication Engineering from the Pt. Ravishankar Shukla University, Raipur Chhattisgarh, India in 2006 and MTech in Electronics from the VJTI, Mumbai Maharashtra, India in 2009. Since 2010, he has been with the Electrical Engineering Department at the Veermata Jijabai Technological Institute (VJTI), Mumbai, India, where he is currently working as a Lecturer and Research Scholar. His research interests include biomedical signal processing, image processing, data analysis, and IOT.

R.N. Awale received his BE and ME in Electronics Engineering from the Marathwada University, Aurangabad, India in 1990 and 1994, respectively. He received his PhD from the SRTM University, Aurangabad, India in 2004. Since 1998, he has been in the Electrical Engineering Department at the VJTI Mumbai, India, where he is currently working as a Professor. He has authored and co-authored more than 70 research papers in peer-reviewed journals and 
conferences. His research interests include a cross-layer design for ad hoc wireless networks, RF, and microstrip antenna and biomedical signal processing. He had filed several patents.

\section{Introduction}

Presently deep learning is a very effective and powerful technique for pattern classification, image tracking, text and scene recognition. Convolutional neural networks, autoencoder, and Boltzmann machine are some different architecture in deep learning. Among all the different architecture, CNN gives high performance on image and pattern classification (Song et al., 2018).

Facial muscles play an important role to give knowledge regarding information passing and message transmitting. The EMG signal always has features such as short amplitude, low frequency and random in nature (Smolka et al., 2017). This is nothing a record of electrical potential generated by facial muscles cells (Ryan et al., 2010). Electrical potentials which are generated by facial cells mainly depend on the physiological properties of face muscles (Haag et al., 2004). Facial emotion is considered as a source of information to understand an effective state of a subject consideration (Huang et al., 2004). The facial electromyogram (FEMG) illustrate information including emotion and muscle fatigue, could disclose out from the face. Facial emotion disorders are a prior sign in various diseases, for example, the stroke patients may feel the swallowing problems in the primary stage (Huang et al., 2004). Many prosthetic devices are supervised and controlled in this way and hence the FEMG gives important information and direction towards biomedical applications and clinical diagnosis (Jiang et al., 2015).

FEMG signals are grouped by non-stationary signals and their study is too complicated (Reaz et al., 2006). Literature shows noise reduction, filtration, segmentation, feature extraction, and classification are the essential stages for analysis of FEMG signals (Ang et al., 2004; Perusquia-Hernandez et al., 2015).

In early days fast Fourier transform (FFT) and parametric power spectrum methods were used for the analysis of biomedical signals (Rezazadeh et al., 2009). These types of transformation methods provide good frequency resolution but suffer from noise sensitivity and spectral losses. The short time Fourier transform (STFT) method provide better resolution in a short window of time for all frequencies. FFT, AR, STFT do not have the time and frequency resolution at the same time (Doulah et al., 2014).

To overcome the problems a powerful tool that is a wavelet transform introduced (Zhang, 2010). The different statistical features are extracted using wavelet coefficients are root mean square (RMS), standard deviation (SD), energy, entropy, etc. which were used for the analysis of FEMG signals (Ang et al., 2004; Duhan et al., 2011). For classification FEMG data-sets, many approaches have been analysed, i.e., artificial neural network (ANN), support vector machine (SVM), K-nearest neighbours (KNN), and FCM, etc. (Rajasekaran and Varghese, 2014; Oskoei and Hu, 2008; Mahyar et al., 2011; Hamedi et al., 2018). Some other techniques which were based on pattern recognition such as fuzzy, probabilistic, principal component analysis, neural networks, the least 
square SVM, etc. (Liejun et al., 2009; Rajasekaran and Varghese, 2014; Hamedi et al., 2015; Jerritta et al., 2014).

The advance signal processing method with the combination of artificial intelligence improves the performance of FEMG signal analysis. The unique features extracted from various classes of kurtogram (different emotion classes) and these features are introduced to the classifier for emotion recognition. Therefore, in this work, a novel technique of kurtogram and deep learning-based FEMG signal analysis technique for emotion classification is proposed. The kurtogram is an estimation of dispersion, represent time-frequency pattern and gives extra information regarding frequency content present in signals (Dwyer, 1983). Kurtogram acts as a feature set for the proposed work and is given as an input to the proposed CNN architecture for emotion detection. The CNN model has analysed effectively in the field of computer vision and signal/image classification (Ren et al., 2017). CNN model has also shown good performance in biomedical related research (Liu et al., 2017; Hosseini et al., 2017).

\section{Materials and methods}

\subsection{Subjects and experiment setup}

In this research work, ten healthy subjects including, five males and five females (ages between 20-35) were selected. Before acquiring FEMG signals, all volunteers were asked to be relaxed and feel true emotions during recording.

Table 1 Involvement of facial muscles responsible for different facial expression

\begin{tabular}{lc}
\hline Facial emotion & Muscles involved \\
\hline Happiness & Orbicularis oculi \\
& Zygomaticus major \\
Anger & Corrugator supercilii \\
& Levator palpebrae \\
Surprise & Frontalis \\
& Levator palpebrae \\
Disgust & Levator labii superioris \\
& Levator labii superioris \\
Sadness & Frontalis \\
& Corrugator supercilii \\
\hline
\end{tabular}

FEMG signal acquisition mostly based on the orientation of fibres with reverence to the electrodes and skin resistance (Thulkar and Hamde, 2015). The myon made wireless device kit is used for the recording FEMG signal (Smolka et al., 2017). This is a two-channel wireless signal acquisition device controlled with bio-feedback software.

At the starting of the experiment, the volunteer was asked to take place in a chair comfortably and the surface electrodes were placed. Figure 1 shows the location of electrode placement in facial muscles. Table 1 depicts the involvement of facial muscles responsible for different facial expression (Chen et al., 2015). The electrode was placed within a distance of $0.5 \mathrm{~cm}$ to $1 \mathrm{~cm}$. After that signal acquisition device was checked and aligned. 
Figure 1 Face muscles responsible for facial expression and location of electrode placement (see online version for colours)

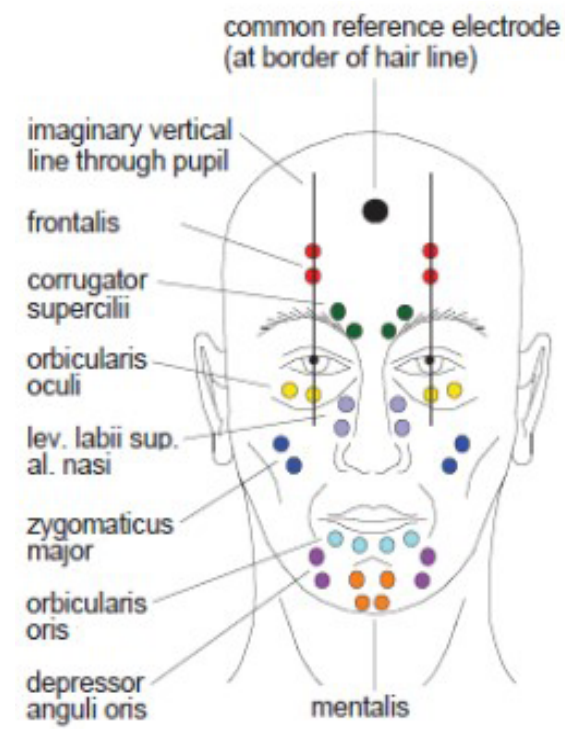

\subsection{FEMG signal acquisition protocol}

Den Broek et al. (2006) have proposed a protocol for facial EMG signal acquisition of different emotion classes. The same protocol has been followed in this work.

Four video clips were selected based on emotional content. The duration of each video clip was 120 seconds taken from current affairs, YouTube channels, and movies. After placing of the surface electrode, the subjects were presented with these selected video sequences, each sequence was presented only once. It was presented to all the subjects, each starting with a different video clip. In between two video sequences a plain blue screen was presented for 30 seconds of duration.

Figure 2 The real-time setup for recording FEMG (see online version for colours)
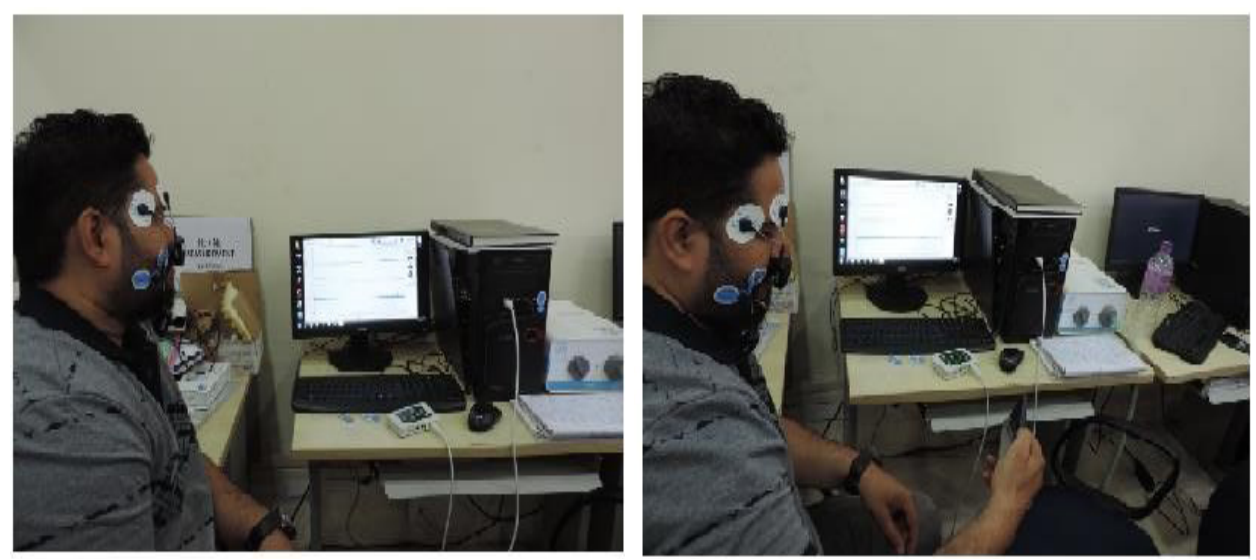
The FEMG signals were acquired by two-channels wireless device, resulting datasets in two-dimensional for each emotion class. Therefore, two datasets for each emotion class has recorded for each person who performed the experiment. Signals were recorded for a sampling frequency of $2 \mathrm{KHz}$. Figure 2 shows the real-time recording of FEMG signals.

\section{Kurtogram}

Spectral kurtogram is a time-frequency representation of non-stationary signal has been introduced by Dwyer (1983) using STFT. Kurtogram of any non-stationary signal is defined by $k(n)$, and it is given by:

$$
k(n)=\int_{-\infty}^{+\infty} G(m, t) e^{j 2 \pi t m} d Y(t)
$$

where $G(n, f)$ is a time-varying transfer function and complex envelope of $k(n)$.

Kurtogram analysis is very useful for analysing non-stationary signals with multi-rate filter bank structure. For each frequency band of FEMG signals its kurtogram pattern is determined.

Figure 3 Kurtogram structure

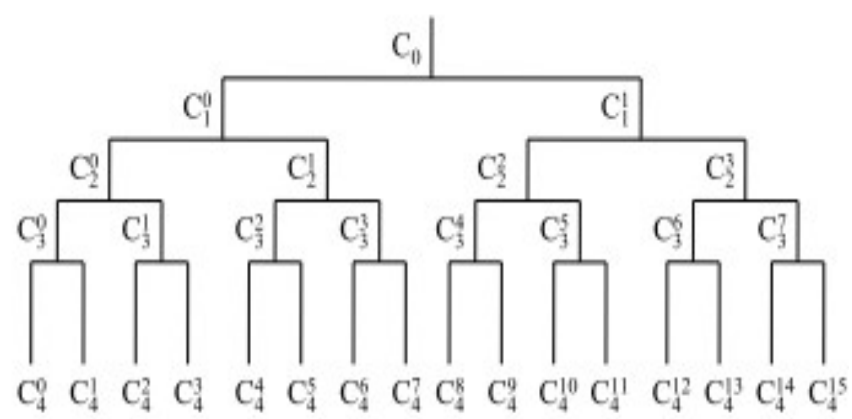

Figure 3 represent the kurtogram structure and Figure 4 illustrates the kurtogram pattern. Maximum level kurtogram gives good accuracy as it effectively contains distinct frequency information for different emotion classes. Figure 5 depicts the FEMG signal of Happiness emotion dataset with its different levels of kurtogram.

Figure 4 Kurtogram pattern (see online version for colours)

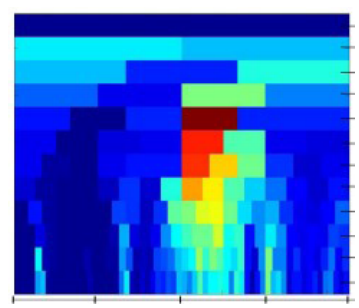


Figure 5 (a) FEMG signal of happiness emotion dataset and its kurtogram at (b) level 1,

(c) level 2, (d) level 3, (e) level 4 and (f) level 5 (see online version for colours)

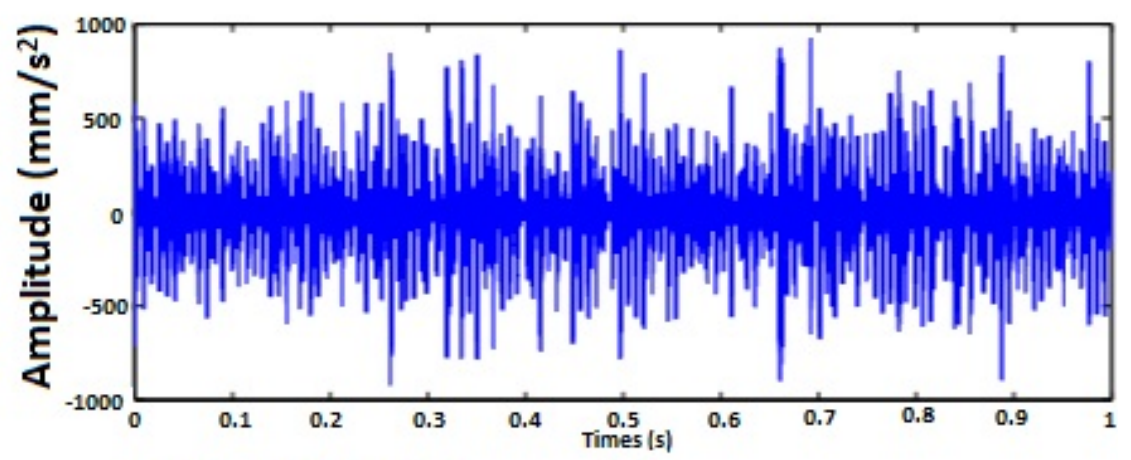

RAW FEMG Signal

(a)

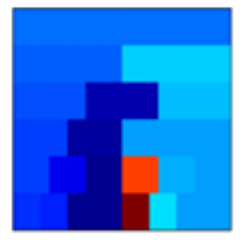

(b)

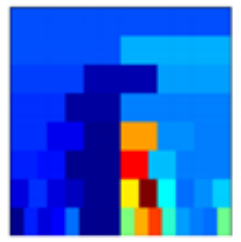

(c)

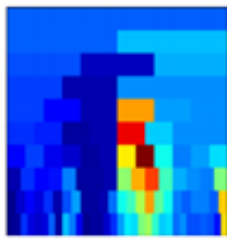

(d)

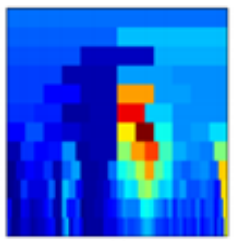

(e)

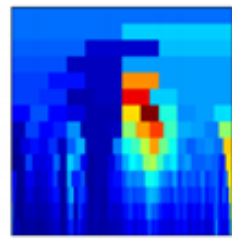

(f)

\section{Convolutional neural network}

Recently, convolutional neural networks $(\mathrm{CNN})$ are the most effective technique to solve problems based on signal and image classification. The CNN architecture assumes that inputs, which allows encoding some properties into the $\mathrm{CNN}$ architecture. To define the boundaries between classes during classification is still difficult.

The classification accuracy is mainly identified by multiple structures with overlapping and non-isolated classes.

The classified class is defined by its degree of belonging to dissimilar classes. This approach is acceptable in different classification related problems and also gives a simple description of the complex feature space. A model architecture of CNN for emotional classification using the kurtogram image of FEMG signal is proposed in the paper.

\subsection{The proposed structure of $C N N$}

The proposed CNN architecture is built up of three different layers. These layers are convolution, sub-sampling, self-organisation with fully connected layers. To design a complete $\mathrm{CNN}$ architecture, we break architecture into three portions. The first part is the combination of convolution layers with sub-sampling layers. The self-organisation layer is the second part of CNN architecture and the last part is a classifier with some fully-connected layers. The proposed architecture of CNN is depicted in Figure 6. 
Figure 6 The proposed architecture of the CNN (see online version for colours)

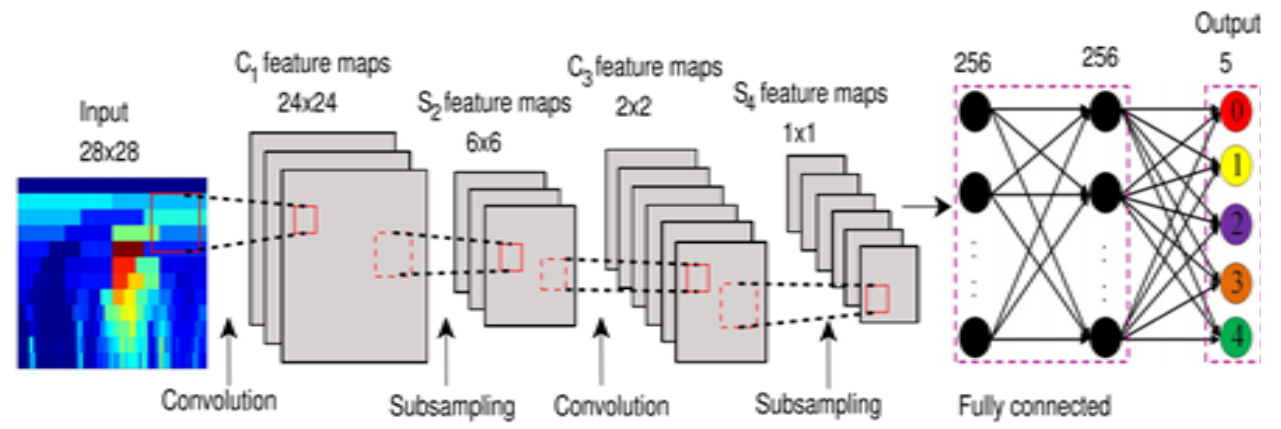

The last part of the architecture is a kind of pre-processor (self-organisation layer). It is situated before fully connected layers. The convolution layer (part 1) receives an input Kurtogram pattern and forms some high-level properties of it by a series of convolution and pooling layers interchange. The structure of the last part of the proposed architecture is shown in Figure 7.

Figure 7 The structure of the last part of $\mathrm{CNN}$

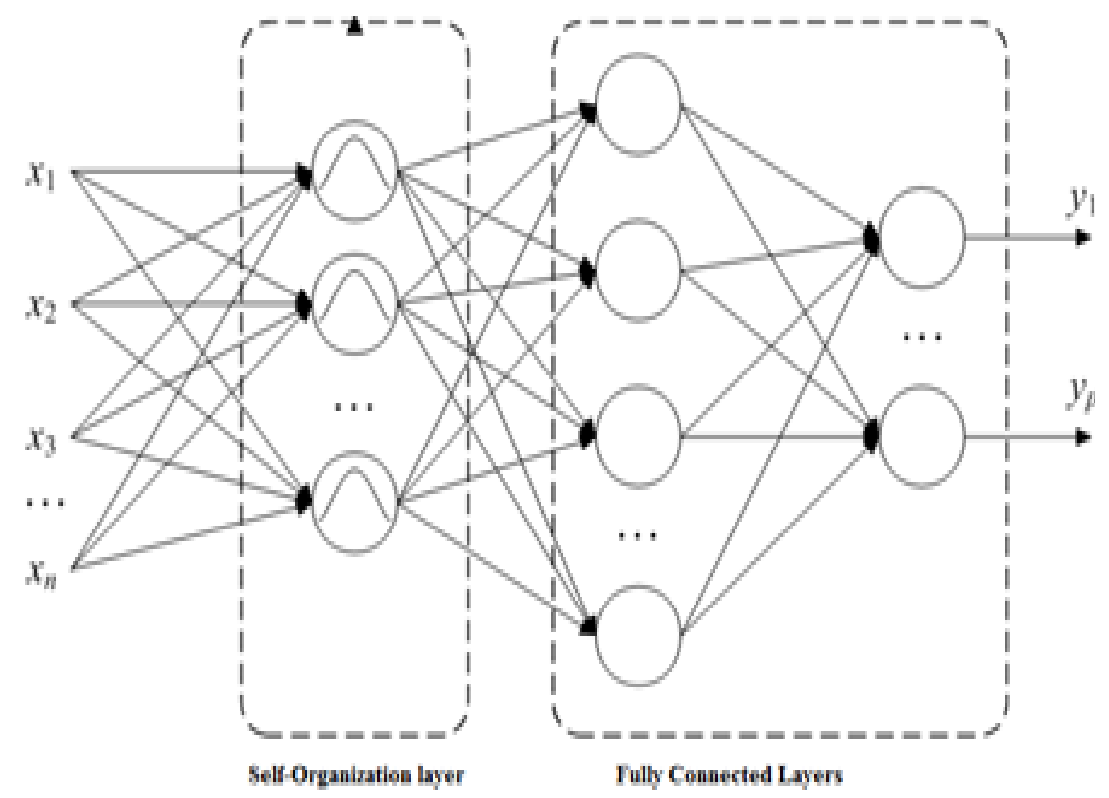

Let $P$ represent the numbers of neuron in self-organised layers. The neurons of the 'self-organised layers' are usually a Gaussian function shown in equation (2):

$$
f(y)=\frac{1}{2 \sqrt{2 \pi}} e^{\frac{(y-n)^{2}}{2 \rho^{2}}}
$$

In equation (2), $n$ is centred of a cluster whereas $\rho$ parameter is a cluster boundary level. If the input vector $y=\left[y_{1}, y_{2}, y_{3} \ldots y_{m}\right]$. The 'self-organised layers' which formed by a 
vector consisting of degrees of belonging y to the precise cluster: $\left[\mu_{1}(y), \mu_{2}(y) \ldots \mu P(y)\right]$. The components $\mu P(y)$ are calculated by the equation (3) which also satisfy the condition in equation (4) for each training sample vector $y(z)$ where $z=1,2,3, \ldots, Z$ where $Z$ represents a number of vectors in the training set.

$$
\begin{aligned}
& \mu_{1}\left(y^{(z)}\right)=f(J)=f\left(\sum_{s=1}^{m} y_{s}^{(z)}\right) \\
& \sum_{p=1}^{P} \mu_{1}\left(y^{(k)}\right)=1
\end{aligned}
$$

The output of the self-organised layers is used as input to the fully connected layers which works as a classifier. The operation of CNN architecture is split in-to three levels. In the first level, the input kurtogram pattern enters through a series of transform actions formed a vector of high-level characteristics. In the second level, the self-organised layer effectuates an initial allocation of input data-sets into precise clusters. In the last level, fully connected layers complete the classification of input datasets, by allocating the result class label to each group of clusters. Figure 7 shows the structure of the last part of CNN.

\section{Results and discussion}

In this paper, we have acquired FEMG datasets from a two-channel wireless data acquisition device. There are five groups of FEMG datasets, contains datasets for five different emotion classes. Each group contains 20 datasets, so the total number of datasets are $100(5 * 2 * 10)$. After pre-processing the segmented FEMG data containing 20,000 samples, used to determine its different level of kurtogram pattern. The block diagram of the proposed work is shown in Figure 8.

Different levels of kurtogram pattern of each FEMG class have been analysed. The selection of the correct kurtogram pattern is based on the classification efficiency of $\mathrm{CNN}$. The CNN architectures are trained to produce efficient results for emotion classification. The performance of $\mathrm{CNN}$ is also compared with other methods, i.e., SVM, $\mathrm{KNN}$, and ANN.

There are five inputs (FEMG kurtogram pattern) each of $1 \times 32 \times 32$ with kernel size $5 \times 5$ has been considered for experimental purposes. The proposed CNN architecture is trained on the different kurtogram patterns to test the adaptability of a model for the various class of FEMG. The performance of the proposed model was done by taking 15 trials, and its average values were reported. A simulation environment consists of i5-CPU with a frequency of $2.6 \mathrm{GHz}, 6.0 \mathrm{~GB}$ RAM, and Ubuntu operating system. Figure 9 shows the experimental results of the CNN architecture gives $93 \%$ of classification accuracy.

For further analysis with SVM, ANN and KNN, the CNN architecture with accuracy is greater than $93.0 \%$ for all the FEMG dataset. The role of the classifier is to identify different sets of data based on training sets and gives correctly classified results. The accuracy of classification depends on the strength of the features. Missing features and outlines in training data can reduce the accuracy of the classifier. A nonlinear type SVM classifier model has used for further analysis. Table 2 compares the different classifier 
results analysis. Table 3 analyses different research works and results in this particular area.

Figure 8 Block diagram of proposed work (see online version for colours)

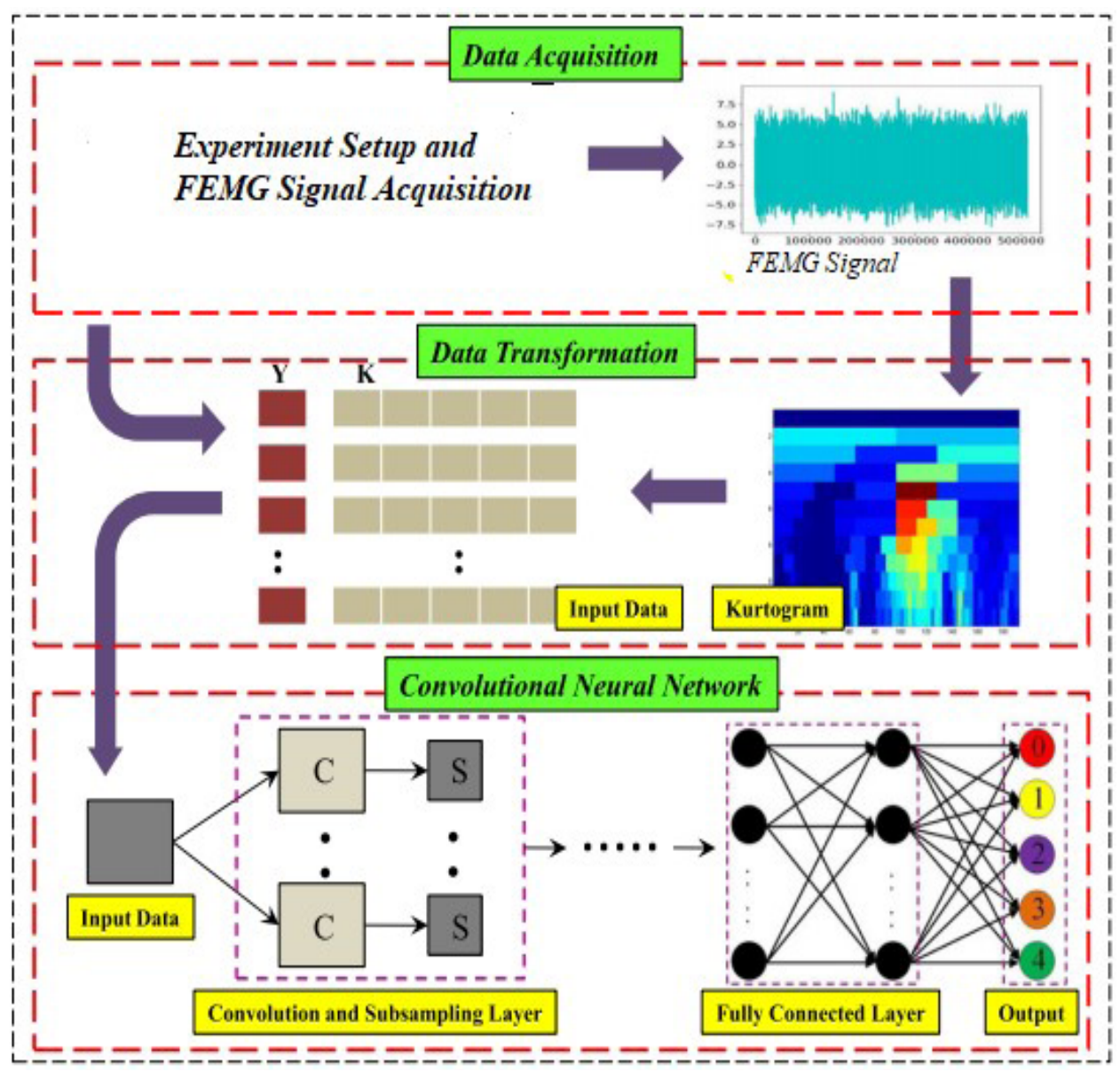

Table 2 Comparison of SVM, ANN, KNN and CNN results (\%)

\begin{tabular}{lcccc}
\hline Parameters & SVM & $A N N$ & $K N N$ & $C N N$ \\
\hline Accuracy (\%) & 81 & 91.66 & 90 & 93 \\
Sensitivity (\%) & 95 & 100 & 100 & 100 \\
Specificity (\%) & 87.5 & 87.5 & 87.5 & 90 \\
Precision (\%) & 65.51 & 80 & 66.67 & 83.33 \\
F-ratio (\%) & 77.55 & 88.8 & 80 & 90.9 \\
\hline
\end{tabular}


Figure 9 Confusion matrix of CNN architecture gives $93 \%$ of classification accuracy with ROC plot (see online version for colours)
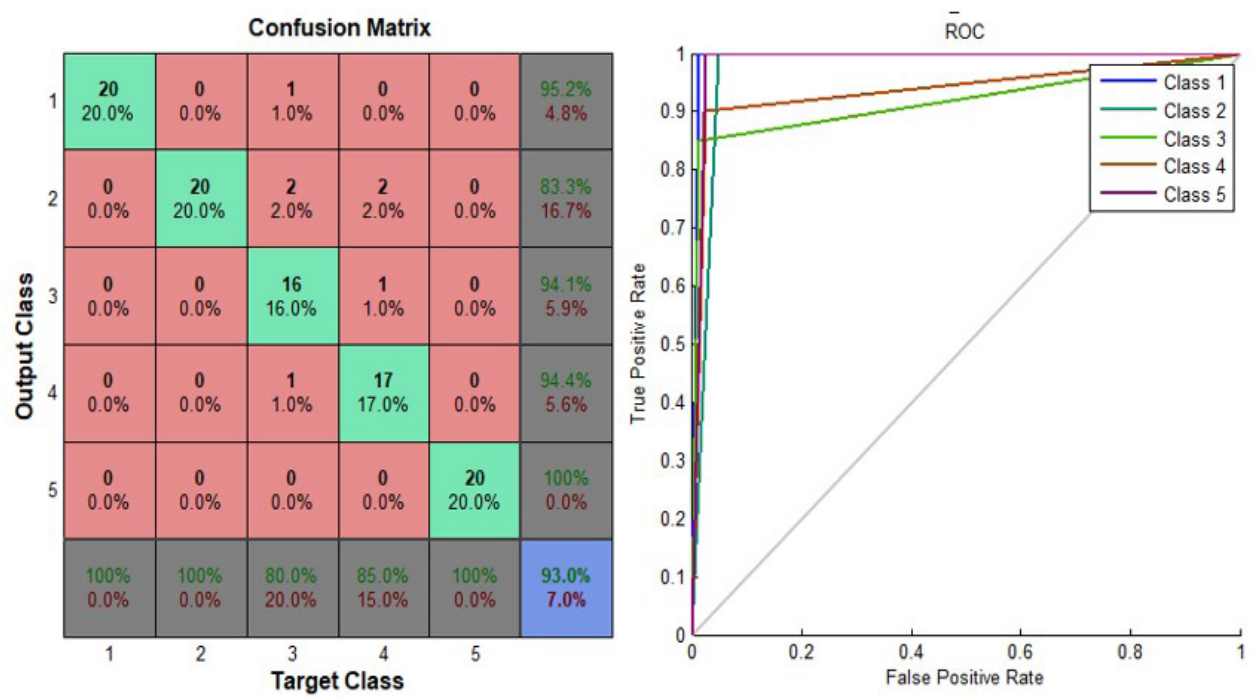

Table 3 Summary of some of the facial expression databases classification

\begin{tabular}{|c|c|c|c|c|c|}
\hline References & $\begin{array}{c}\text { No. of } \\
\text { participants }\end{array}$ & $\begin{array}{c}\text { No. of } \\
\text { channels }\end{array}$ & $\begin{array}{l}\text { Types of } \\
\text { classifier }\end{array}$ & $\begin{array}{l}\text { Selected } \\
\text { features }\end{array}$ & $\begin{array}{l}\text { Classification } \\
\text { result }\end{array}$ \\
\hline $\begin{array}{l}\text { Rezazadeh et al. } \\
\text { (2009) }\end{array}$ & 3 & 3 & FCM & RMS & $92.6 \%$ \\
\hline $\begin{array}{l}\text { Ang et al. } \\
(2004)\end{array}$ & 2 & 2 & $\begin{array}{l}\text { Minimum } \\
\text { distance }\end{array}$ & $\begin{array}{c}\text { RMS, } \\
\text { MEAN, PDS }\end{array}$ & $94.44 \%$ \\
\hline $\begin{array}{l}\text { Hamedi et al. } \\
\text { (2018) }\end{array}$ & 10 & 3 & SVM, FCM & RMS, BTFD & $80.4 \%$ and $91.8 \%$ \\
\hline $\begin{array}{l}\text { Sinha and } \\
\text { Parsons (1996) }\end{array}$ & 27 & 2 & LDA & $\begin{array}{l}\text { Statistical } \\
\text { features }\end{array}$ & $86 \%$ \\
\hline Current study & 10 & 2 & $\begin{array}{l}C N N, S V M, \\
A N N, K N N\end{array}$ & Kurtogram & $\begin{array}{c}93 \%, 81 \% \\
91.66 \% \text { and } 90 \%\end{array}$ \\
\hline
\end{tabular}

\subsection{SVM results}

SVM is a supervised learning machine based on statistical theory. SVM gives good accuracy, as well as better predictors on a large number of datasets hence it is normally used in FEMG signal analysis (Oskoei and $\mathrm{Hu}, 2008$ ). SVMs can classify the data which linearly as well as nonlinear separated in a feature space. In this method, feature vectors are the series of predictor values which reside closest to decision boundaries that classify classes.

Selecting the best location of decision hyperplane is actually a big issue of optimisation, where independent kernel functions are implemented to design the linear boundaries through mapping. The selection of the kernel function and its parameter is very important things in SVM. 
There are various kernel functions are available such as linear, quadratic, polynomial, Gaussian radial basis, etc. (Oskoei and $\mathrm{Hu}, 2007$; Sharma and Veer, 2016). The mathematical expression for the polynomial kernel is given below:

$$
N p\left(y, y_{i}\right)=\left(y * y_{i}+1\right)^{d}
$$

The polynomial kernel function for SVM has selected after various trails and error basis. Equation (5) shows the mathematical expression of the polynomial kernel, where $d$ is the parameters related to the polynomial kernel. Here the selection of the proper value of kernel parameter $\mathrm{d}$ is important because it affects the performance of a classifier. The incorrect and improper value of $\mathrm{d}$ leads to various problems such as over and underfitting, which causes incorrect classification accuracy. Several values of $d$ have tried and based on that the value of $d$ has selected, which gives the best classification accuracy. Finally, the classifier has designed with a polynomial kernel function.

Here the polynomial kernel with poly order $3(d=3)$ gives $81 \%$ accuracy compared to the others nonlinear kernel. Cross-validation type-hold out ' $80-20$ ' type has been implemented. Figure 10 shows the SVM confusion matrix representing $81 \%$ of classification accuracy with ROC plot.

Figure 10 SVM confusion matrix representing $81 \%$ of classification accuracy with ROC plot (see online version for colours)
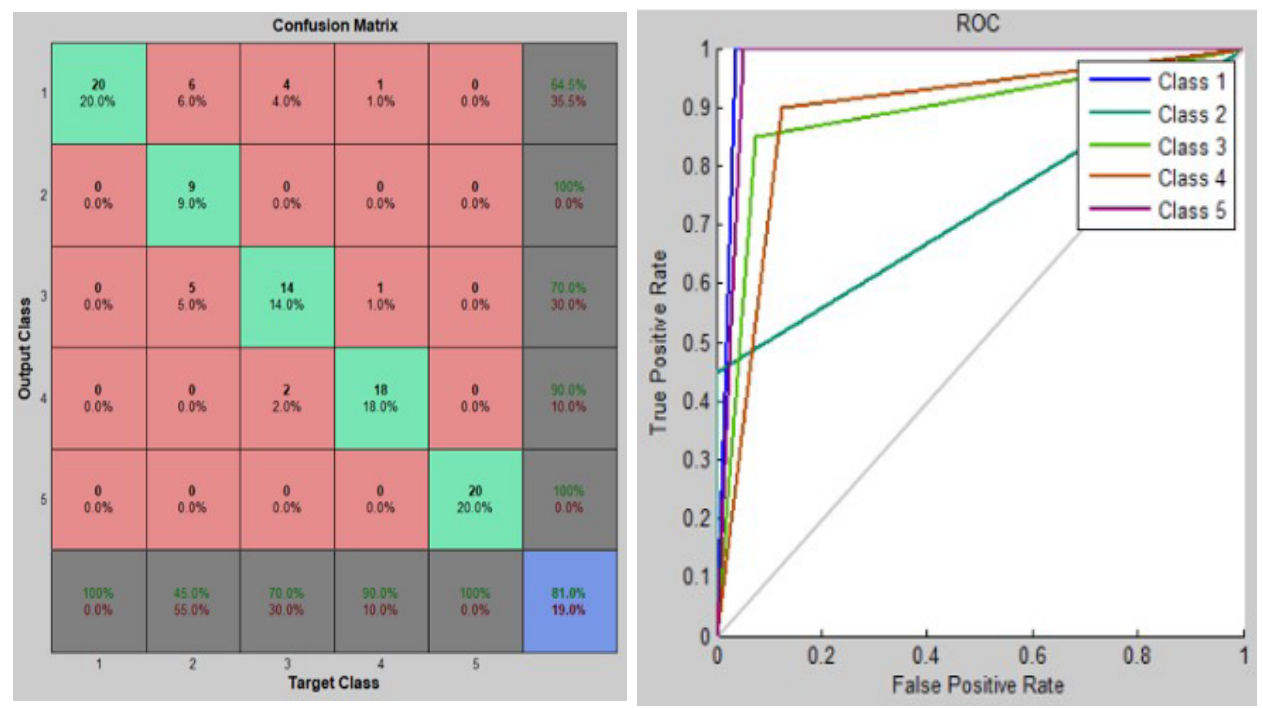

\section{Conclusions}

This study presents a novel approach to recognise different facial gestures. FEMG signals have been recorded from ten different subjects. The proposed model of CNN architecture for kurtogram pattern classification to recognise different facial emotion is presented in the paper. The proposed approach combines the kurtogram analysis with $\mathrm{CNN}$ architecture and is capable of controlling impreciseness. Kurtogram is a powerful technique for analysis of random data-sets and provides more information regarding 
different frequency bands. Different levels of the kurtogram pattern have been used for experimental work to measure the efficiency of $\mathrm{CNN}$ architecture. Based on training and testing accuracy results, the proposed technique provides better accuracy in less training time as compared to other classifiers results. 93\% classification accuracy was achieved, which positively proved the success of our proposed method. Therefore, it can be concluded that spectral kurtogram analysis with $\mathrm{CNN}$ architecture is a useful method to represent the characteristic of FEMG signals. To acquire good results, various architectures of CNN are considered with different levels of the kurtogram pattern. The analysis concludes that the classification accuracy can be increased by increasing the level of kurtogram.

For further analysis with SVM, ANN, and KNN, statistical features are extracted using the Daubechies family ( $\mathrm{db} 8$ ) of the wavelet. The performance of ANN classifier is reasonably better than SVM and KNN classifier since it has a good recognition rate.

\section{References}

Ang, L.B.P., Belen, E.F. et al. (2004) 'Facial expression recognition through pattern analysis of facial muscle movements utilizing electromyogram sensors', in TENCON, IEEE Region 10 Conference, Vol. C.

Chen, Y., Yang, Z. and Wang, J. (2015) 'Eyebrow emotional expression recognition using surface EMG signals', Neuro Computing, November, Vol. 168, No. C, pp.871-879, DOI: 10.1016/j.neucom.2015.05.037.

den Broek, E.L., van Herk, J. et al. (2006) 'Computing emotion awareness through facial electromyography', in Huang, T.S. et al. (Eds.): Computer Vision in Human-Computer Interaction, ECCV 2006, Lecture Notes in Computer Science, Vol. 3979, Springer, Berlin, Heidelberg.

Doulah, A.B.M.S.U., Fattah, S.A. et al. (2014) 'Wavelet domain feature extraction scheme based on dominant motor unit action potential of EMG signal for neuromuscular disease classification', in Proceedings of the Eighteenth International Conference on Artificial Intelligence and Statistics, AISTATS 2015, IEEE Trans. Biomed. Eng., deeply supervised nets, San Diego, California, USA, 9-12 May 2015, Vol. 8.

Duhan, M. et al. (2011), 'Study of signal processing techniques for EMG analysis', Int. J. of Biomechatronics and Biomedical Robotics, Vol. 1, No. 3 pp.141-148.

Dwyer, R. (1983) 'Detection of non-Gaussian signals by frequency domain kurtosis estimation', in International Conference on Acoustics, Speech, and Signal Processing (ICASSP'83), IEEE, Vol. 8, pp.607-610.

Haag, A., Goronzy, S., Schaich, P. and Williams, J. (2004) 'Emotion recognition using biosensors: first steps towards an automatic system', Affective Dialogue Syst.

Hamedi, M., Salleh, S., Ting, C., Astaraki, M. and Noor, A. (2018) 'Robust facial expression recognition for MuCI: a comprehensive neuromuscular signal analysis', in IEEE Transactions on Affective Computing, Vol. 9, No. 1, pp.102-115, DOI: 10.1109/TAFFC.2016.2569098.

Hamedi, M., Salleh, S-H. and Noor, A.M. (2015) 'Facial neuromuscular signal classification by means of least square support vector machine for MuCI', J. Soft Computing, May, Vol. 30, No. C, pp.83-93, DOI: 10.1016/j.asoc.2015.01.034.

Hosseini, M., Pompili, D., Elisevich, K. and Soltanian-Zadeh, H. (2017) 'Optimized deep learning for EEG big data and seizure prediction BCI via the internet of things', IEEE Trans Big Data, Vol. 3, No. 4, pp.392-404.

Huang, C-N., Chen, C-H. and Chung, H-Y. (2004) 'The review of applications and measurements in facial electromyography', Journal of Medical and Biological Engineering, Vol. 25, No. 1, pp.15-20. 
Jerritta, S., Murugappan, M., Wan, K. and Yaacob, S. (2014) 'Emotion recognition from facial EMG signals using higher-order statistics and principal component analysis', Journal of the Chinese Institute of Engineers, Vol. 37, No. 3, pp.385-394.

Jiang, M., Rahmani, A., Westerlund, T., Liljeberg, P. and Tenhunen, H. (2015) 'Facial expression recognition with sEMG method', in 2015 IEEE International Conference on Computer and Information Technology; Ubiquitous Computing and Communications; Dependable, Autonomic and Secure Computing; Pervasive Intelligence and Computing LIVERPOOL, pp.981-988, UK.

Liejun, W., Xizhong, Q. and Taiyi, Z. (2009) 'Facial expression recognition using improved support vector machine by modifying kernels', Inform. Technol. J., Vol. 8, No. 4, pp.595-599.

Liu, W., Wang, Z., Liu, X., Zeng, N., Liu, Y. and Alsaadi, F.E. (2017) 'A survey of deep neural network architectures and their applications', Neuro Computing, Vol. 234, pp.11-26 [online] http://bura.brunel.ac.uk/handle/2438/14221.

Mahyar, H., Salleh, S-H. et al. (2011) 'Surface electromyography-based facial expression recognition in bi-polar configuration', Journal of Computer Science, Vol. 7, No. 9, pp.1407-1415, Science Publications, ISSN: 1549-3636 (C) 2011.

Oskoei, M.A. and Hu, H. (2007) 'Application of support vector machines in upper limb motion classification using myoelectric signals', Proceedings of the IEEE International Conference on Robotics and Biomimetics, IEEE Xplore Press, Sanya, 15-18 December, pp.388-393.

Oskoei, M.A. and Hu, H. (2008) 'Support vector machine-based classification scheme for myoelectric control applied to upper limb', IEEE Trans. Biomed. Eng.

Perusquia-Hernandez, M., Hirokawa, M. and Suzuki, K. (2015) 'Spontaneous and posed smile recognition based on spatial and temporal patterns of facial EMG', in 2017 Seventh International Conference on Affective Computing and Intelligent Interaction (ACII), San Antonio, TX, USA, pp.537-541.

Rajasekaran, M.P. and Varghese, S.M. (2014) 'EMG signal classification using ANN and ANFIS for neuro-muscular disorders', Int. J. of Biomedical Engineering and Technology, Vol. 16, No. 2, pp.156-168.

Reaz, M.B.I., Hussain, M.S. and Mohd-Yasin, F. (2006) 'Techniques of EMG signal analysis detection, processing, classification and applications', Biol. Proced., Vol. 8, No. 1, pp.11-35, Published online 23 March, DOI: 10.1251/bpo115, URL: BiolProced Online.

Ren, S., He, K., Girshick, R. and Sun, J. (2017) 'Faster-CNN: towards real-time object detection with region proposal networks', IEEE Trans. Pattern Anal. Mach. Intell., Vol. 39, No. 6, pp.1137-1149.

Rezazadeh, I.M., Wan, X., Wang, R. et al. (2009) 'Toward affective handsfree human machine interface approach in virtual environments-based equipment operation training', 9th International Conference on Construction Applications of Virtual Reality, 5-6 November.

Ryan, A., Kohn, J.F., Lucey, S., Saragih, J. and Lucey, P. et al. (2010) 'Automated facial expression recognition system', Proceedings of the IEEE International Carnahan Conference on Security Technology, IEEE Xplore Press, Zurich, 5-8 October.

Sharma, T. and Veer, K. (2016) 'Wavelet analysis-based evaluation of electromyogram signal using human-machine cooperation', Int. J. of Medical Engineering and Informatics, Vol. 8, No. 4 pp.317-328.

Sinha, R. and Parsons, O.A. (1996), 'Multivariate response patterning of fear', Conference on Cognition and Emotion, Vol. 10, No. 2, pp.173-198.

Smolka, J., Zurek, S., Lukasik, E. and Skublewska-Paszkowska, M. (2017) 'Heart rate estimation using an EMG system integrated with a motion capture system', International Conference on Electromagnetic Devices and Processes in Environment Protection with Seminar Applications of Superconductors (ELMECO \& AoS), Lublin, pp.1-4. 
Song, C., Chen, C., Li, Y. and Wu, X. (2018) 'Deep reinforcement learning apply in electromyography data classification', 2018 IEEE International Conference on Cyborg and Bionic Systems (CBS).

Thulkar, D. and Hamde, S.T. (2015) 'Facial electromyography for characterization of emotions using LabVIEW', IEEE International Conference on Industrial Instrumentation and Control (IClC).

Zhang, X. (2010) 'Wavelet transform theory and its application in EMG signal processing', Seventh International Conference on Fuzzy Systems and Knowledge Discovery. 